\title{
QUELQUES ASPECTS DU BILINGUISME DANS LA ZONE LINGUISTIQUE ROUMAINO-SERBO-CROATE*
}

En parlant de ce problème nous allons souligner - en premier lieu l'influence serbo-croate sur la langue roumaine (et non pas 1'influence inverse), 1 parce que nous avons examiné la zone qui était longtemps sous l'influence de la langue serbo-croate. 2 La zone qui nous intéresse pour 1'instant, c'est le Banat, c'est-à-dire le côté yougoslave de cette région, tandis que de l'autre côté, en Banat Roumain, on rencontre une situation différente. Précisons qu' ici nous n'allons pas traiter des problèmes du bilinguisme en Serbie, au sud du Danube, où nous avons affaire avec une problématique spéciale. 3 Comme nous avons déjà délimité la zone de l'intérêt linguistique que nous allons examiner, il faut d'abord expliquer les conditions historiques dans lesquelles se sont développés les procès linguistiques.

* Ces pages contiennent le texte intégral de notre communication présentée en langue serbo-croate au 1er Congrès de Linguistique Appliquée de Yougoslavie, qui a eu lieu à Belgrade du 16 au 18 décembre 1977.

1 A propos de 1'influence de la langue roumaine sur les parlers serbes du Banat Yougoslave cfr. M.D. Savić, Interferenţe linguistice (sîrbo-române) la Alibunar ['Interférences linguistiques (serbo-roumaines) à Alibunar'] (dans Lumina XX, Pančevo 1968, t. 1-3, p. 103-112).

2 En préparant le présent exposé nous $n$ 'avons pas tenu compte de la différence existant entre 1'influence de la langue serbo-croate standard et celle des parlers serbes de la dite zone, considérant avoir examiné tant le language parlé que la langue écrite d'une couche de personnes bilingues. Quant aux détails, nous référons à M.D. Savić, Uticaj srpskog jezika na rumunske govore jugoslovenskog Banata [' $L$ ' influence de la langue serbe sur les parlers roumains du Banat Yougoslave'] (dans Contributii la istoria culturală a românilor din Voivodina $I$, Zrenjanin 1973, p. 75-92).

3 Quant à la zone au sud du Danube, cfr. M.D. Savić, Alcuni influssi serbi sulle parlate valacche della Serbia Orientale (dans Actes du XII e Congrès International de Linguistique et de Philologie Romane, avril 1968, Bucarest 1971, II, p. 1069-1076); Dve zajedničke crte u srpskim i vlaškim govorima ['Deux caractéristiques communes dans les parlers serbes et valaques'] (dans Analele Societăţii de limba română a PSA Voivodina, 3-4, 1972-73, p. 509-518); et Neke jezičke podudarnosti u srpskom i rumunskom narodnom pesništvu istočne Srbije ['Quelques concordances linguistiques dans les chants populaires serbes et roumains en Serbie Orientale'] (dans Radovi Simpozijuma II, Pančevo 1974, p. 537-546). 
Sans doute, 1'ancienne symbiose slavo-romane, soit en Dacie ou dans les Balkans, s'est transformée peu à peu dans cette zone en une symbiose serbo-roumaine, grâce à la même religion qui avait rattaché les deux différents groupes linguistiques, ce qui était très important dans certaines époques historiques. 4 La religion commune symbolisait en même temps 1'appartenance à la même culture, ce qui se reflétait dans la langue commune de culture. Soulignons que 1'ancienne langue slave fonctionnait longtemps officiellement dans 1'Eglise et dans l'administration des Principautés Roumaines, ce qui a rendu possible plus tard la soumission des sujets des deux peuples parlant une des deux langues différentes à la juridiction de l'Eglise Métropolitaine Orthodoxe de Sremski Karlovci.

Au début de notre siecle A. Meillet a remarqué que les innovations dans le lexique, c'est-à-dire dans le domaine sémantique des mots, expriment en premier lieu les conditions sociales. 5 Il est donc clair que les parlers roumains du Banat Yougoslave, étant toujours en contact avec la langue serbo-croate, étaient soumis à l'influence d'une certaine manière de vivre de la population de cette zone. Il ne faut pas oublier que nous sommes ici à la périphérie de 1'aire linguistique roumaine, dans une zone qui ne fut jamais irradiée d'un centre linguistique et culturel roumain, ce qui a conféré une note spéciale à ces parlers. Presse, radio, télévision, cinéma, théâtre, ainsi que tous les moyens de communication $y$ ont un caractère régional, différent de ce que possède la langue roumaine moderne, enrichie d'innovations et de néologismes.

4 Cfr. Radu Flora, Despre comunitatea ierarhică sîrbo-română sub jurisdictia Mitropoliei din Sremski Karlovci si despre importanta arhivelor mitropolitane pentru studierea legăturilor sîrbo-române din trecut ['Sur la communauté ecclésiastique serbo-roumaine sous la juridiction de. la Métropolie de Sremski Karlovci et sur 1'importance des archives métropolitaines pour étudier les relations serbo-roumaines dans le passé'] dans le livre Relaţi sîrbo-române - noi contributii, Pančevo 1968, p. 204-229; et Nikola Gavrilović, Rumunski jezik u administraciji Karlovačke mitropolije u XVIII veku ['La langue roumaine dans 1'administration de la Métropolie de Sremski Karlovci au cours du XVIII e siècle'] (dans Radovi Simpozijuma III, Zrenjanin 1977, p. 115-122).

5 Antoine Meillet, Comment les mots changent de sens, dans 1'Année sociologique (1905-1906), republie dans Linguistique historique et linguistique générale, Paris 1948, p. 230 ss. 
En cette zone-là nous rencontrons, outre aux personnes parlant les deux langues différentes, ceux qui pratiquent les deux variantes d'une même langue. 6 Selon les remarques faites de R. Flora qui a examiné sur une longue période les particularités de la langue roumaine de la zone en question, il s' agit de parlers périphériques. ${ }^{7}$ Ces parlers ne disposent pas de néologismes qui sont nombreux dans la langue roumaine littéraire et qui font partie aussi d'une langue standard, c'est-à-dire ils se rapprochent des parlers serbo-croates, se conformant à l'exigence de la langue serbo-croate littéraire qui évite les innovations et les néologismes.

$N^{\prime}$ étant pas en mesure de traiter toutes les particularités d'influence de la langue serbo-croate sur les parlers de diverses localités à population roumaine du Banat Yougoslave, nous allons nous borner à discuter le phénomène de cet aspect linguistique qui nous est le plus accessible, cette possibilité nous étant offerte par L'Atlas linguistique du Banat Yougoslave de R. Flora (nous remercions 1' auteur d'avoir mis à notre disposition son oeuvre précieuse). 8 D'un côté, nous allons étudier les exemples dépouillés dans la presse de cette zone, publiée en langue roumaine et, de l'autre côté, les résultats d'une enquête faite sur les étudiants bilingues qui fréquentent les cours de langue roumaine à la Faculté de Philologie de Belgrade.

6 Nous envisageons les termes "bilinguisme" et "diglossie". Cfr. à cet égard Michele Famiglietti, Il bilinguismo in una comunità italo-albanese e proposte didattiche (dans Rassegna Italiana di Linguistica Applicata VII, $2-3,1975$, p. 103-123). L' affirmation que l'auteur y présente ne peut être appliquée que partiellement à la zone en question. Quant à la diglossie, qui est typique, comme nous 1'avons remarqué a plusieurs reprises, du sentiment linguistique d'un grand nombre de Roumains vivant en Banat Yougoslave, nous pensons que ce problème pourra être éclairci par la méthode de Lorenzo Milani, quoiqu'il discute d'une aire bien différente. Cfr. Chiamo uomo chi è padrone della sua lingua, dans le livre de L. Renzo M. Cortelazzo, La lingua italiana oggi: un problema scolastico e sociale, Bologna, 1977, p. 39-53.

7 Cfr. R. Flora, Rumunski banatski govori u svetlu lingvističke geografije ['Les parlers roumains banatiens à la lumière de la géographie linguistique'], Beograd 1971, passim.

8 Le titre roumain de cette oeuvre est Atlas linguistic al Banatului iugoslav. 
L'influence de la langue serbo-croate est bien visible dans les domaines lexical et syntaxique, tandis que la phonétique et la morphologie sont plus stables. 9

L'influence serbo-croate est bien évidente dans certaines formes de calques linguistiques:

- calques des locutions verbales et leurs applications au lieu des constructions roumaines, qui font partie intégrante de la langue roumaine administrative et officielle: "Noul program de doi ani prevede ca sa se intensifice si dezvolte si mai mult ..." (5. II) ${ }^{\mathbf{1 0}}$ au lieu de "prevede intensificarea si dezvoltarea";

- calques de quelques expressions fixes ou syntagmes: "... considerăm că hotărîrea adusă la întrunirea de la Alibunar ..." (5. II) au lieu de "că propunerea facuta";

- calques lexicaux: "... i-au organizat o asteptare maiestoasă ..." (28. XI) au lieu de "i-au organizat o primire";

- calques des régences verbales d'après celles qui sont typiques de syntaxe serbo-croate: "... care va dezbate asupra acestor probleme" (28. XI) au lieu de "va dezbate aceste probleme"; "Peste 720 de mii de persoane au subscris împrumut" (15. I) au lieu de "la împrumut" ou "împrumutului";

- calques analogues aux syntagmes de base existants dans la langue serbo-croate: "a vizita concerte" (29. I) au lieu de "a frecventa concerte" ou "a merge la concerte"; "... cu scopul de a-i şoca pe ascultători" (29. I) au lieu de "de a-i impresiona"; "întreprinderea ' 6 oktobar' felicită tuturor cetățenilor" (28. XI) au lieu de "pe toţi cetăţenii"; "aşa e în natura noastră a voivodenilor" (28. XI) au lieu de "aşa e natura noastră"; "noi astăzi, introducem în viaţă un nou sistem" (5. XI) au lieu de "traducem în viaţa";

9 Outre l'article déjà cité Uticaj srpskog jezika na rumunske govore jugoslovenskog Banata, nous mentionnerons encore M.D. Savić, Palatalizacija i umekšavanje u rumunskim govorima jugoslovenskog Banata ['Palatąlisation et amouillement dans les parlers roumains du Banat Yougoslave? (dans Radovi Simpozijuma III, Zrenjanin 1977), où 1' auteur pense qu'il est impossible attribuer quelques-unes des particularités phonétiques des parlers roumains du Banat Yougoslave à l'influence de la langue serbo-croate, parce que leur zone très vaste renferme aussi une aire qui ne fut jamais sous l'influence de la langue serbo-croate.

10 Tous les exemples cités sont dépouillés dans le journal Libertatea, Pančevo, de l'an 1977. 
- calques des compléments temporels: "în anul trecut" (29. I) au lieu de "anul trecut"; "î̀ săptămîna viitoare" (29. I) au lieu de "săptămîna viitoare"; "în timp de cinci luni" (29. I) au lieu de "timp de cinci luni";

- calques des prépositions: "cînd a fost desemnat de cel mai bun jucător" (29. I) au lieu de "drept cel mai bun"; "banii strînşi în formă de cotizatie" (29. I) au lieu de "sub formă de ...";

- remplacement de la diathèse en vigueur dans la langue roumaine par celle qui existe en serbo-croate: "De data aceasta ne vom reţine asupra unui singur obiectiv" (29. XI) au lieu de "vom retine un singur obiectiv"; "ocazia să işi facă cunostinta cu unele forme ale muzicii ..." (29. I) au lieu de "să facă ...".

En relation avec ces calques faut-il faire mention encore du genre des substantifs roumains qu' on change souvent d'après le genre des substantifs serbo-croates: "nu se poate înmagazina nici o boabă, care are o cantitate mai mare de umezeală ..." (29. I) au lieu de "bob (de grîu)"; "... de adversară avînd formaţia locală ..." (25. I) au lieu de "drept adversar".

Si nous trouvons dans ces parlers des néologismes verbaux empruntés récemment à la langue roumaine, ils ne sont pas conjugués d'après les régles de la grammaire roumaine: "hîrtie de lucru în care se rezumează" (28. XI) au lieu de "se rezumă"; "Pentru soluţionarea acestei probleme este necesar să se investeze un miliard si 400 milioane de dinari ..." (28. XI) au lieu de "să se investească".

Un éloignement particulier des règles fondamentales imposées par la syntaxe roumaine est évident dans 1'emploi de l'article, qui est - comme il est bien connu - postposé. Etant sous l'influence de la langue serbo-croate qui ne possède pas d'article, les personnes bilingues, n'ayant pas le sentiment propre de cette catégorie linguistique, ne se servent ni de l'article défini ni de 1'indéfini, soit en langue colloquiale où son emploi est inévitable, soit aussi parfois en langue écrite: "... este usor să ţii discurs ..." (28. XI) au lieu de "un discurs": "Dragostea si datorie faţă de cupă" (29. XI) au lieu de "datoria"; "... în viitor devin adevărată mică olimpiadă" (29. XI) au lieu de "o adevărată".

L'influence linguistique du serbo-croate se manifeste aussi dans l'emploi des formes pronominales de politesse, qui - à la différence de la langue serbo-croate qui est plus pauvre à cet égard - sont nombreuses en roumain et confèrent à cette langue une nuance spéciale, p. ex. : dumneavoastră, dumneata, dumnealui, dînsul, dînsa. Même les personnes bilingues qui ont séjour né longuement en Roumanie évitent les formes mentionnées, et séduites par les parlers serbes locaux en Yougoslavie, se rattachent, étant entre deux systemes linguistiques différents, à celui qui existe en serbo-croate, utili- 
sant la forme voi 'vous', quoiqu' elle ne soit pas conforme aux exigence fondamentales de la langue roumaine traditionnelle et standard. ${ }^{11}$

Quant au système morphologique, qui présente la partie la plus stable de chaque langue, il reste presque invariable, ce qui explique comment la plupart des mots empruntés à travers la langue serbo-croate s'est assimilée au système roumain: "finea" au lieu de "finele", "istoriat" au lieu de "istorie", "despărţămînt" au lieu de "secţie", "paraliză" au lieu de "paralizie"; "... muzicenii italieni la prima probă" (25. I) au lieu de "repetiţie"; "avea o frică de neînchipuit faţă de paraliză" (29. XI) au lieu de "paralizie".

Quand il s'agit de mots nouveaux ou d'expressions créées grâce au progrès scientifique et technique, les personnes bilingues les traduisent en se référant à l'exemple proposé par la langue serbo-croate ou les empruntent directement (à la langue d'origine): "... construirea unei mori de slad" (25. I) au lieu "de melasð"; "... a primit un honorar de 400 mii de dolari" (25. I) au lieu de "onorariu"; "... se produce bere luminoasă ..." (25. I) au lieu de "bere blondă"; ".. exportăm 30 de procente ..." (25. I) au lieu de "30 la sută".

Comme nous avons pu le constater, le nombre des mots empruntés à la langue serbo-croate qui se rencontrent dans les parlers roumains du Banat Yougoslave dépasse de beaucoup celui qui peut être enregistré dans la presse roumaine locale. Les preuves à cet égard nous sont fournies par quelques textes dialectaux recueillis sur le terrain et en premier lieu par le langage quotidien des étudiants bilingues en question. 12 Quelques-uns des étudiants enquetés déclarent employer, dans des monologues intérieurs et en songe, une des deux langues différentes, ce qui dépend du milieu roumain ou serbo-croate, où ils se trouvent à ce moment-là. Naturellement, notre constation se réfère à quelques personnes, et non pas à tout le groupement dont nous discutons. Nous avons aussi constaté, p. ex., que la personne qui s'explique mal ou à peine en roumain an début, commence, après avoir lu quel-

11 A l'égard de ce probleme cfr. Lia Magdu, Abateri de la normele limbii literare - practicată la noi in Voivodina ['Eloignements des règles prescrites par la langue littéraire - pratiqués chez nous en Voivodine'] (dans Contributii la istoria culturalä a romanilor din Voivodina III, Zrenjanin 1976 , p. 157-167), une contribution qui s'occupe de quelques particularités du bilinguisme que nous $n$ 'avons pas traité dans notre communication d' aujourd' hui.

12 Nous portons une attention particulière à un certain nombre de textes dialectaux que R. Flora a publiés dans son livre Dijalektološki profil rumunskih banatskih govora sa Vršačkog područja ['Le profil dialectologique des parlers roumains banatiens dans la zone de Vršac'], Novi Sad 1962. 
ques pages du texte roumain, à parler très bien, ce qui veut dire que les bases scientifiques d'une langue se réduisent à la connaissance de sa grammaire et sa structure.

On constate en même temps que les personnes bilingues parlant le roumain et le serbo-croate connaissent les deux langues mieux que ceux qui parlent deux langues du même groupe (p. ex., dans la zone balkanique, le serbo-croate et le macédonien ou le serbo-croate et le bulgare etc.). Cela est dû au fait que les personnes parlant les deux langues apparentées, confondent instinctivement leurs structures linguistiques et lexicales, ce qui les empêche de parler couramment chacune de ces deux langues. 13 sous ce rapport on peut expliquer aussi des nombreux cas de négligence inconsciente des règles stabilisées dans la langue roumaine, soit qu'il s'agisse d'utiliser des prépositions inadéquates: ". . . a fost singurul motiv din care am procedat astfel" (28. XI) au lieu de "pentru care", 14 soit qu'il s'agisse d'employer les adjectifs possessifs qui ne correspondent pas à l'esprit de la langue roumaine: "şi pentru că este vorba de filmul amatoricesc" (29. XI) au lieu de "de amatori", ou d'une série d'autres inconséquences.

Ces tendances peuvent parfois modifier le sens, p. ex.: "reactivarea amatorismului cultural" (5. II) au lieu de "reactivarea muncii culturale de amatori", particulierèment parce que le substantif amatorism possède en roumain une nuance injurieuse.

Conformément aux tendances dont nous avons fait mention et en particulier parce que le style administratif, officiel et journalistique de cette région s'avère très imperméable aux néologismes, il apparait très simplifié et plein de répétitions à une personne instruite d'origine de l'aire centrale de la langue roumaine. Considérons quelques-unes des particularités mentionnées qui font une impression très déplaisante sur le sentiment linguistique du Roumain habitué au style contemporain déterminé et à la langue standard:

- répétition: "să amintim propunerea Organizatiei femeilor din Uzdin care propune o colaborare mai amplă, mai bogată şi mai organizată a Organizatiei femeilor ..." (28. XI);

- absence de néologismes, ce qui réduit la façon de s'exprimer à la simplification: "unicul prilej de distractie pentru săteni este dansul organizat ... (5. II);

13 Cfr. M. C. Galli et G. Harrison, Il bilinguismo 'zoppo' degli Albanesi d'Italia dans le livre déja cité La lingua italiana oggi ..., p. 425-438.

14 Quand il s'agit du langage parlé des Roumains de Voîvodine, il faut observer que quelques prépositions y ont prévalu (en qualité de calque) sous l'influence serbo-croate, ce qui est rarement visible dans la langue écrite. 
- répétition des mêmes prépositions, ce qui rend monotone le discours ou le texte: "... produse din concentrate din import" (28. XI) au lieu de "din concentrate importate"; "... un turneu de tenis de masă" (28. XI) au lieu de "la tenis de masă".

Même si l'on utilise des néologismes qui font partie intégrante de la langue roumaine contemporaine, leur emploi ne suit pas toujours les règles stylistico-lexicales: "... să preîntîmpine Republica cu ploaie şi inima curată" (28. XI) au lieu de "să intîmpine", tenant compte que le verbe a preîntîmpina signifie "prévoir les mesures pour empêcher quelque chose de mauvais".

L'ordre des mots, bien des fois, ne suit pas les topiques roumains, mais ceux qui sont en vigueur en serbo-croate, p. ex.: "Ştił̧i voi să citił̧i?" (28. XI) au lieu de "Voi ştiţi să citiţi?"; "Ingheţuri se vor înregistra în orele de dimineaţă" (5. II) au lieu de "î̀n orele de dimineaţă se vor înregistra îngheturi".

L'absence du sujet de la phrase dans le style journalistique, ce qui est dû sans doute à une traduction effectuée vite et de façon inconséquente de la langue serbo-croate, est remplacée par la forme impersonnelle du prédicat: "În discuţiile care au durat peste două ore, au examinat situaţia actuală" (5. II) au lieu de "s-a examinat".

On rencontre aussi la négligence de l'infinitif dans la langue écrite, c'est-à-dire dans le style administratif et officiel, où l'infinitif est toujours remplacé par les constructions subjonctivales. D' autre part, 1'emploi de 1'infinitif en qualité d'objet direct présente dans la langue roumaine contemporaine une expression régulière et officielle du style bureaucratique.

Dans la langue roumaine contemporaine le vocatif des substantifs de genre féminin tend à être identifié avec le nominatif, tandis que les parlers roumains en Voïvodine continuent à employer la désinence o sous l'influence directe du serbo-croate.

On est frappé aussi par l'emploi inadéquat des adjectifs en rapport avec les substantifs auxquels ils se réfèrent, tenant compte des règles qui sont en vigueur dans la langue roumaine standard, où l'adjectif suit le substantif. S'il précède le substantif, il lui confère une nuance spéciale et en même temps sert à mettre en évidence une signification particulière. Quant aux parlers roumains de Voïvodine, dans la plupart des cas, 1'adjectif y précède le substantif $d$ 'après le modèle existant en serbo-croate, mais - nous pouvons aussi le constater - il reste sans I'expressivité qu'il possède dans la langue roumaine standard. De toute façon, il s'agit d'une influence régressive du point de vue des langues romanes, tandis que le serbo-croate a à sa disposition d'autres moyens qui servent à mettre en évidence l'attribut. 
Une caractéristique commune de la langue des Roumains du Banat Yougoslave (liée tant au langage parlé qu'à son aspect écrit) est la tendance à eviter l'emploi du futur antérieur qui est remplacé par le présent, tandis que 1'ambiguité temporelle est levée en ajoutant des adverbes de temps: mîine, luna viitoare, etc. 15

En conclusion, si nous voulions indiquer en peu de mots les motifs qui ont rendu possible (et qui continuent à le faire encore aujourd' hui) l'influence de la langue serbo-croate sur les parlers des Roumains de Voivodine (sans tenir compte de raisons générales qui peuvent influencer les personnes bilingues d'autres langues parlées dans notre Pays), nour devrions quitter les cadres strictement linguistiques et prendre en considération aussi des facteurs extralinguistiques, p. ex., sociaux et ethnographiques, dont nous citons quelques exemples:

- la population parlant la langue roumaine n'est pas conservatrice à l'égard des coutumes, des croyances et de la tradition;

- relations de mariage et de parenté entre Serbes et Roumains, auxquelles a contribué aussi l'appartenance à la même religion, ce qui était très important dans le passé;

- on n'empêche pas les jeunes gens de parler serbo-croate en famille, mais, au contraire, ils y sont encouragés;

- dans de nombreux villages et localités la population est hétérogène du point de vue démographique.

En terminant notre exposé, nous sommes obligés de souligner que nous n'avons réussi mettre en évidence que quelques-unes des particularités linguistiques des Roumains bilingues en Vö̈vodine. Il reste encore un nombre de phénomènes caractéristiques que nous n'avons pas précisés et qui méritent d'être enregistrés et examinés, ce qui devra être réalisé. Cependant,

15 Soulignons que le système verbal roumain du langage parlé en Banat Yougoslave est très simplifié $s^{\prime}$ il $s^{\prime}$ agit des formes temporelles, étant influencé par celui de la langue serbo-croate, dans laquelle les catégories temporelles sont remplacées par l'aspect verbal. Cfr. M.D. Savić, Cum se reflectă aspectul verbal sî́rbocroat în limba română? ['Comment se reflete l'aspect verbal serbo-croate dans la langue roumaine?'] (dans Analele Societăţii de limba română a PSA Voivodina 2, Zrenjanin 1971, p. 25-39) et Iskazivanje prošlosti u srpskohrvatskoj i rumunskoj publicistici [' $L$ 'expression du temps passé dans les publications journalistiques serbo-croate et roumaine'] (dans Radovi Simpozijuma I, Pančevo 1972, p. 369-382). 
nous proposons à ceux qui s'occuperont de cette tâche de tenir compte aussi de l'âge, de l'éducation, du sexe des personnes enquêtées et de l'ambiance où elles vivent. 16

\section{Rezumat}

\section{UNELE ASPECTE ALE BILINGVISMULUI ÎN ZONA LIMBILOR ROMÂNĂ ŞI SÎRBOCROATĂ}

Observaţiile autorilor se bazează pe cercetarea fenomenului de bilingvism în zona Voivodinei si se sprijină pe exemple concrete luate din publicaţiile oficiale de expresie românească şi pe anchetarea studenţilor bilingvi care frecventează cursurile de limba română la Universitatea din Belgrad.

Influîenta limbii sîrbocroate este simţită î̀ special în domeniul lexical şi sintactic, partea morfologică şi fonetică a lịmbii fiind mai stabilă.

Cea mai frecventă particularitate lingvistică este cea a calchierii sub diferite aspecte: -folosirea construcţiilor verbale în locul celor nominale; calchierea regimului verbelor, a anumitor sintagme, prepoziţii, a diatezei; schimbarea genului substantivelor după cel din sirbocroată; folosires incorectă a determinării proclitice prin articol; absenţa pronumelui de politeţe; împrumutarea cuvintelor noi datorate progresului tehnico-ştiinţific, din sîrbocroată, etc.

16 Outre I'autre littérature scientifique que nous avons eu à notre disposition en préparant cette communication, nous avons utilisé aussi les oeuvres suivantes: Veroboj Vildomec, Multilingualism, Leyden 1963; Theodor Elwert, Das zweisprachige Individuum (dans Abhandlungen der Geistes- und sozialwissenschaftlichen Klasse der Akademie der Wissenschaft und der Literatur, Mainz 1960); Uriel Weinreich, Languages in Contact, London-Hag-Paris 1967; Bertil Malberg, Encore une fois le substrat (dans Studia linguistica XVII, 1963); August Kovačec, Bilingvizam i aloglotski utjecaj na morfosintaktičku strukturu jezika (na istrorumunjskom materijalu) ['Le bilinguisme et 1'influence alloglottique sur la structure morphosyntaxique d'une langue (en base des matériaux istro-roumains)'] (dans Suvremena lingvistika, nr. 4: Jezici u kontaktu i lingvističko posudjivanje, Zagreb 1967, p. 101-114); R. Flora, Aria sudică a graiurilor româneşti (sub semnul unei influente puternice a graiurilor sîrbeşti) ['L' aire du midi des parlers roumains (exposés à une influence puissante des parlers serbes'] dans le livre déjà cité Relaţii sîrbo-române ..., p. 374-378. 
Bilingvii folosesc în discursul interior sau in vis una din limbile pe care o utilizează curent în perioada respectivă. De asemenea, vorbitorii a două limbi diferite, cum sînt sîrbocroată şi română, cunosc în mai mare măsură cele două ideomuri faţă de alţi bilingvi cu limbi înrudite (sîrbocroată şi bulgară, sîrbocroată şi macedoneană, etc.). Autorii explică aceasta prin structura apropiată şi fondul lexical înrudit ale celor două limbi. Vorbitorii fac astfel instinctiv asocieri si amestecuri, care defavorizează înşusirea corectă a limbilor.

Factorii sociali si etnografici care au dus la puternica influentă a limbii sîrbocroate asupra graiului românesc din PSA Voivodina ar fi, după părerea autorilor, următorii:

- populatia vorbitoare de limba română nu este conservatoare în obiceiuri, tradiţii si credinţe;

- întemeierea familiilor se face adesea prin înrudirea cu populaţia sîrbă;

- tinerei generaţii nu $i$ se impun interdicţii în folosirea limbii sîrbocroate în familii, dimpotriva copii sînt stimulaţi s-o folosească;

- asezarea gospodariilor în vatra satelor se face pe un spatiu nedelimitat, compoziţia demografică fiind eterogenă. 